\title{
Lenguajes para el aprendizaje
}

\author{
Languages for Learning
}

\section{Alejandra Meneses A. ${ }^{1}$, Evelyn Hugo R. ${ }^{1}$ y Paola Uccelli ${ }^{2}$}

${ }^{1}$ Pontificia Universidad Católica de Chile, Facultad de Educación

${ }^{2}$ Harvard Graduate School of Education

\section{Resumen}

\begin{abstract}
Con frecuencia se oponen las prácticas académicas a las vernáculas; sin embargo, desde una aproximación sociocultural y pragmática, se ha demostrado que el lenguaje se desarrolla a lo largo de la escolaridad a partir de la participación de los niños, niñas y jóvenes en diferentes contextos comunicativos permeables e interdependientes. Este número temático incluye nueve artículos que desarrollan relaciones entre lenguajes y aprendizajes -más allá de la dicotomía «dentro y fuera de la escuela»-. Desde diversas perspectivas teóricas y metodológicas, las investigaciones abordan los lenguajes situadamente, con énfasis en el registro, en el género discursivo o en el campo disciplinar. Algunos estudios destacan el aprendizaje del lenguaje verbal desde lo receptivo o lo productivo; otros, las interacciones entre modalidades y modos semióticos para la construcción de diversos géneros. Asimismo, los artículos conceptualizan el aprendizaje de maneras diferentes. Se argumenta que el desarrollo del lenguaje sirve de precursor y continúa como proceso evolutivo conjunto y crucial para el aprendizaje de la lectura y escritura. Algunos proponen que el conocimiento del lenguaje es un facilitador del aprendizaje, ya que subrayan el potencial epistémico de la lectura y escritura como herramienta de reflexión. También se amplía el estudio del aprendizaje más allá de la institución escolar, pues se investiga acerca de prácticas culturales en espacios digitales. Los estudios presentan resultados que aportan al campo del desarrollo del lenguaje y de su enseñanza, y tienen implicancias para la práctica pedagógica, ya que explicitan el rol de los lenguajes en la construcción de los aprendizajes, desde la valoración de la diversidad lingüística, contextual y cultural.
\end{abstract}

Palabras clave: lenguaje, aprendizaje, literacidad, multimodalidad, géneros discursivos, registros

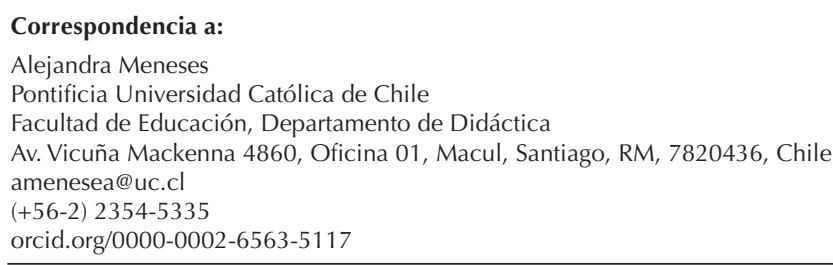




\section{Abstract}

Academic practices often come into conflict with vernacular practices. However, from a sociocultural and pragmatic approach, it has been shown that language develops throughout education due to the children and young people's participation across different permeable and interdependent communicative contexts. This thematic issue includes nine papers that examine the relations between languages and learning — going beyond the simple dichotomy of "in-school and out-of-school". From a diversity of theoretical and methodological perspectives, the studies address languages situated, emphasizing the register, the discursive genre, or the disciplinary field. Some of the studies highlight verbal language learning from the receptive or productive perspective, while others focus on the interactions between modalities and semiotic modes for the construction of different genres. The papers also conceptualize learning in different ways. It is argued that language development serves as a precursor and continues as a coordinated and crucial evolutionary process for learning to read and write. Some of the authors propose that language knowledge facilitates learning, as they underline the epistemic potential of reading and writing as a tool for reflection. The study of learning is also extended beyond the school institution, exploring cultural practices in digital spaces. The papers offer results that contribute to the field of research on language development and its teaching and outline the implications for pedagogical practices since they make the role of languages in the construction of learning more explicit, based on the valuation of linguistic, contextual, and cultural diversity.

Key words: language, learning, literacy, multimodality, discursive genres, registers

La escuela es un espacio sociocultural en el que los aprendizajes se co-construyen a través de la mediación del lenguaje verbal (Grøver, Uccelli, Rowe \& Lieven, 2019; Schleppegrell, 2004; Snow \& Uccelli, 2009) y de su interacción con otros modos semióticos en los que el lenguaje visual juega un rol fundamental (Macken-Horarik, Love, Sandiford \& Unsworth, 2017; Unsworth \& Cleirigh, 2009). De manera simultánea, niños, niñas y jóvenes fuera del espacio escolar participan y co-construyen prácticas de literacidad complejas, mediadas por distintos modos semióticos y medios para alcanzar una amplia variedad de propósitos comunicativos (Barton \& Hamilton, 2000; Buckingham, 2006; Burnett \& Merchant, 2015; Mills, Unsworth \& Exley, 2018).

Si bien se ha tendido a establecer una oposición entre prácticas académicas y vernáculas, desde una aproximación sociocultural y pragmática, la investigación actual ha demostrado que el lenguaje se desarrolla a lo largo de la escolaridad a partir de la participación y la interacción de niños, niñas y jóvenes en diferentes contextos comunicativos, los que son permeables e interdependientes (Berman \& Ravid, 2009; Grøver et al., 2019; Nippold, 2007; Uccelli, 2019). Los géneros discursivos que circulan en distintos contextos (por ejemplo, comentarios sobre memes en redes sociales o discusiones sobre textos de historia) se caracterizan no solo por desarrollar temas específicos, sino también por el uso recurrente de determinados recursos discursivos y lingüísticos para alcanzar propósitos específicos de las comunidades de habla. Por lo tanto, el aprendizaje del lenguaje se entiende como la expansión de la flexibilidad retórica, es decir, la ampliación progresiva de recursos lingüísticos para navegar por una creciente variedad de contextos pragmáticos (Ravid \& Tolchinsky, 2002). Desde esta perspectiva, el aprendizaje del lenguaje continúa desarrollándose de manera importante durante la adolescencia y a lo largo de la vida con la enseñanza de nuevos registros y géneros discursivos que no solo expande los repertorios lingüísticos y discursivos, sino que además potencia las habilidades de reflexión metalingüística para lograr una participación activa y crítica en diversos espacios sociales y culturales (Ravid \& Tolchinsky, 2002; Uccelli, Phillips Galloway \& Qin, 2020). 
Este número temático de Pensamiento Educativo Revista de Investigación Educacional Latinoamericana, surge del interés por reunir estudios en español e inglés que indagaran sobre las relaciones entre lenguaje y aprendizaje en distintos contextos. Los artículos publicados se construyen desde diversas perspectivas teóricas y metodológicas para abrir la discusión sobre los lenguajes para el aprendizaje, desde una mirada que permita repensar la dicotomía «dentro y fuera de la escuela».

Más allá de las distinciones y los énfasis específicos, los estudios comparten una aproximación sociocultural y funcional hacia el lenguaje (Berman \& Ravid, 2009; Ravid \& Tolchinsky, 2012; Snow \& Uccelli, 2009). Este es conceptualizado como un repertorio de recursos discursivos y lingüísticos usados -de manera oral o escritapor hablantes que simultáneamente construyen sentidos, establecen relaciones sociales y negocian identidades a partir de la producción o interpretación de diferentes géneros discursivos.

Asimismo, se reconoce que -desde siempre- las personas han materializado sus discursos no solo mediante la palabra, sino también a través de otros modos semióticos, como el visual; sin embargo, solo en las últimas décadas se ha comenzado a investigar empíricamente el rol de estos otros lenguajes en los espacios de aprendizaje y su relación con el lenguaje verbal (Unsworth \& Cleirigh, 2009). Por lo tanto, en este número temático se abordan los lenguajes en plural, ya que los aprendizajes son co-construidos por la interacción entre diversos modos semióticos. Estos lenguajes también permiten una construcción flexible de significados entre contextos debido a la porosidad de sus bordes, lo que favorece un intercambio y dinamismo entre espacios, más que una división entre lo escolar y lo no escolar.

Este número presenta nueve estudios que se organizan según los niveles educativos. Las investigaciones comprenden desde kínder hasta primer año de universidad, con mayor presencia de los estudios vinculados con la lectura en educación primaria y con mayor atención a la escritura en los estudios enfocados en secundaria.

El estudio de Silva y Jéldrez analiza las preferencias por libros narrativos o informativos de una muestra de niñas y niños chilenos en dos momentos del inicio del aprendizaje formal de la lectura: kindergarten y primero básico. Este estudio ofrece evidencia que cuestiona la arraigada idea de que al inicio del desarrollo de la lectura la preferencia va siempre dirigida a libros narrativos. Se detectó en kindergarten una preferencia significativa por textos informativos sobre los narrativos, mientras que en primero básico la preferencia fue balanceada. Este artículo subraya la relevancia de que tanto los libros narrativos como los informativos formen parte del repertorio disponible para las y los niños que se están iniciando en la lectura si se quiere atender a sus intereses. Asimismo, las autoras enfatizan en la necesidad de que las y los niños estén en contacto con textos informativos desde los primeros ańos para familiarizarse tempranamente con el lenguaje de estos textos vinculados fuertemente con el aprendizaje en distintas disciplinas (por ejemplo, textos de ciencias y de historia), que presentan recursos lingüísticos y discursivos que los distinguen de textos narrativos y cuyo aprendizaje es importante en la transición a los grados más avanzados.

El artículo de Taboada Barber, Vizcaya y Lutz explora la contribución de la Teoría de la Mente (ToM por su sigla en inglés) -habilidad sociocognitiva que permite explicar la conducta de las personas a partir de sus creencias, estados mentales y deseos- a la comprensión oral y lectora en inglés de estudiantes bilingües emergentes entre $3^{\circ}$ y $5^{\circ}$ grados en Estados Unidos. Los resultados muestran que una mayor habilidad en ToM se correlaciona de manera moderada y significativa con la comprensión oral y lectora, la identificación de palabras y el vocabulario. Asimismo, aun después de controlar por grado, vocabulario y decodificación, las diferencias individuales en ToM predicen una proporción pequeña de la comprensión oral y lectora en inglés. Como puntualizan las autoras, este estudio exploratorio ofrece resultados sobre un tema mínimamente explorado con estudiantes bilingües emergentes de origen hispanohablante. Los hallazgos permiten identificar el rol que juegan diferentes habilidades en los procesos de comprensión oral y escrita de estudiantes diversos. Este artículo aporta con evidencia para el diseño de intervenciones que superen aproximaciones desde una teoría del déficit y avancen hacia la valoración 
de la diversidad lingüística. Por otra parte, este estudio aplica un marco que complejiza el modelo simple de la lectura-Simple View of Reading- para lograr detectar predictores de la comprensión lectora que van más allá de destrezas básicas y conocimiento del lenguaje en lectores bilingües emergentes.

Romero-Contreras, Silvia-Macedo y Snow exploran la contribución de dos factores lingüísticos -vocabulario y habilidades de lenguaje académico- al aprendizaje de la lectura y la escritura en las áreas disciplinares. Su estudio extiende la investigación sobre el lenguaje académico y la comprensión lectora en español con estudiantes mexicanos de fines de primaria e inicio de la secundaria con el fin de aportar a la complejización del modelo del Simple View of Reading. A partir del marco de habilidades clave de lenguaje académico propuesto por Uccelli, Phillips Galloway, Barr, Meneses y Dobbs (2015), el estudio comprueba el poder predictivo de esta constelación de habilidades lingüísticas mediante las cuales se construyen significados en los contextos escolares disciplinares y que operan más allá de la palabra. Los resultados muestran patrones similares, pero contribuciones distintas para los grupos de estudiantes investigados. Las autoras enfatizan el carácter dúctil del lenguaje académico y, por ende, enseñable en los contextos escolares, lo que abre un nuevo punto de entrada para la enseñanza de la literacidad escolar. Asimismo, destacan que el nivel socioeconómico de las y los estudiantes no es un predictor significativo de la comprensión lectora una vez que se introducen el vocabulario y las habilidades de lenguaje académico, lo que contribuye a la conceptualización de estas variables lingüísticas como catalizadores de equidad y de justicia educativa. Este estudio aporta al campo de la literacidad intermedia con datos de estudiantes hispanohablantes, ya que en contextos latinoamericanos la investigación se ha centrado mayormente en la literacidad inicial.

Por su parte, Sánchez y García contribuyen a la discusión sobre la literacidad intermedia mediante la reconsideración de tres estudios experimentales en los que identifican las dificultades de estudiantes para alcanzar la comprensión de textos explicativos disciplinares. Los autores sistematizan cuatro retos que enfrentan los estudiantes en su aprendizaje de la lectura: aprender a leer (decodificar), aprender a comprender, leer para aprender y leer múltiples fuentes para alcanzar una meta. Leer para aprender es desafiante porque las y los estudiantes se enfrentan a textos disciplinares con temáticas abstractas desarrolladas mediante el lenguaje académico escolar. Por lo tanto, lectores aprendices tienen que desarrollar estrategias para desenvolverse en un contexto de alta complejidad. Los autores investigan tres factores para comprender cómo estos influyen en la lectura estratégica: el efecto de claves orales y escritas para apoyar la comprensión de textos explicativos, la distancia al interior del texto entre una clave y la idea a la que esta refiere y, por último, la mantención de un objetivo de lectura en una tarea con múltiples fuentes. El primer estudio destaca el mayor aporte de las claves orales ofrecidas por docentes para andamiar la comprensión lectora en comparación con aquellas solo introducidas en el texto, pero estas últimas son más efectivas que los textos sin ningún tipo de ayuda. En la medida en la que las y los estudiantes tienen mayor dominio del lenguaje académico -o, en términos de los autores, competencia retórica- pueden beneficiarse de igual manera de las claves orales o de las claves escritas. Asimismo, destacan la dificultad que conlleva para estudiantes de $4^{\circ}$ y $5^{\circ}$ grado la interpretación de claves escritas distantes de las ideas que introducen. Por último, demuestran que en un contexto de lectura de múltiples fuentes resulta complejo para estudiantes de $4^{\circ}$ y $5^{\circ}$ grado mantener activo su objetivo de lectura a lo largo de la tarea. De ahí que propongan el enfoque de ayudar a comprender para la lectura de textos disciplinares, pues se ofrecen todas las ayudas orales y escritas -andamios para asegurar el acceso a las ideas- para que las y los estudiantes alcancen la comprensión de un texto en específico.

El artículo de Sepúlveda, Paulet y Cardoso se circunscribe en la línea de intervenciones educativas que proponen una enseñanza explícita del lenguaje para favorecer el aprendizaje disciplinar. Las investigadoras aluden a la extensa tradición que da cuenta de que los textos académicos y disciplinares resultan demandantes para las y los estudiantes en tanto suelen presentar gran densidad nominal, inclusión de términos técnicos, numerosas palabras abstractas y metáforas gramaticales, entre otros (Fang, Schleppegrell \& Cox, 2006; Schleppegrell, 2001). El estudio describe los cambios en las prácticas de dos docentes después de participar en un programa que busca mejorar la lectura y el aprendizaje a partir de textos en el área de Historia con actividades agrupadas 
en cuatro bloques que responden a diferentes finalidades y momentos de interacción con los textos de estudio: 1) preparar a las y los estudiantes para estudiar el texto; 2) leer y comentar el texto; 3) estudiar el texto, y 4) comunicar la comprensión del texto. La investigación se realizó en dos escuelas de Brasil, en aulas de $4^{\circ}$ grado. Los resultados indican que, con la incorporación de las propuestas del programa, las profesoras ofrecieron más oportunidades para atender, recuperar, comentar y analizar el contenido informativo de los textos, al mismo tiempo que aumentó la participación de las y los estudiantes, sobre todo en el aula de la docente que desde un inicio presentaba un estilo pedagógico más interactivo.

El artículo de Resina y Salas examina las relaciones entre la lengua oral y la lengua escrita en el contexto de aprendizaje del discurso argumentativo. Estudiantes bilingües de catalán-español de primer año de secundaria de una escuela en Barcelona fueron divididos en dos grupos: uno recibió primero una intervención en lengua oral (basada en el programa Word Generation) y luego una intervención en lengua escrita (basada en el modelo de SRSD de Graham y Harris, 2009), mientras que el otro grupo recibió intervenciones idénticas, pero administradas en orden inverso. Ambos grupos fueron comparados con otro que solo recibió las clases habituales de la asignatura de Lengua y Literatura Castellana. Los estudiantes fueron evaluados antes del inicio de las intervenciones, al finalizar la primera intervención y al finalizar la segunda; se analizaron los efectos intramodalidad e intermodalidad. Los resultados indican que ambas intervenciones mostraron efectos significativos intramodalidad: la intervención en lengua oral mejoró la calidad del discurso argumentativo oral y del lenguaje académico, y la intervención en lengua escrita produjo una mejora en la calidad y la longitud (medida según el número de palabras) de los textos argumentativos escritos. Ahora bien, los resultados también indican que las y los estudiantes que recibieron primero la intervención en lengua oral escribieron textos de mejor calidad en la primera medición que el grupo control, pero de menor calidad que el grupo que recibió la intervención en lengua escrita primero. Este artículo reafirma la idea de que la investigación en adquisición del lenguaje en la etapa de desarrollo tardío debería atender a las implicaciones de las relaciones bidireccionales entre ambas modalidades, sin dejar de advertir y explicar las especificidades en el aprendizaje de cada modalidad discursiva.

El artículo de Valdivia aborda los aprendizajes alcanzados por jóvenes chilenos a través de Instagram. Su estudio etnográfico plantea que las redes sociales agrietan las fronteras del espacio institucionalizado para el aprendizaje y muestra cómo en los espacios digitales las y los jóvenes construyen sentidos sobre todo mediante la visualidad. La autora caracteriza las prácticas digitales de ocho jóvenes -cinco hombres y tres mujeres- con distintos grados de producción digital en las redes sociales y distingue entre productores esporádicos, activos o avanzados. A partir de la observación de las rutinas juveniles, se concluye que la esfera digital potencia cruces entre las formas de participación del hogar, la escuela y el grupo de pares. El análisis de las producciones digitales muestra distintos grados de dominio y de reflexión sobre los recursos semióticos y retóricos usados por los jóvenes en diversas actividades. Las y los adolescentes más activos en Instagram buscan compartir experiencias personales en las que los sentidos se configuran a partir de las relaciones entre imagen, humor y palabras tanto en inglés como en español. Por su parte, las y los productores avanzados muestran un dominio de conocimientos estéticos y visuales para la creación fotográfica, la que es publicada en cuentas con una línea curatorial definida. De particular interés resultan las producciones de jóvenes que participan en organizaciones feministas, pues sus acciones políticas se materializan discursivamente en las redes sociales. Para posicionarse, las producciones son construidas mediante un lenguaje académico como recurso de legitimación de sus visiones y objetivos para lograr incidir en sus instituciones escolares. Este artículo pone de relieve, por una parte, el carácter fluido de los lenguajes usados en las producciones que circulan en el espacio digital y, por otra, los distintos propósitos de participación social de jóvenes en una plataforma digital y multimodal de alcance global.

Desde la Lingüística Sistémico Funcional, Unsworth propone un marco analítico para la descripción de ensambles multimodales publicados en textos escolares de Ciencias, así como aquellos creados por estudiantes australianos de secundaria. El autor observa que se ha estudiado más la tecnicalidad y agregación de significados en 
los textos verbales, lo que da cuenta de la complejidad lingüística a la que se enfrentan los estudiantes. Sin embargo, se han explorado mínimamente estas características en los ensambles multimodales usados para la construcción del conocimiento científico escolar. La propuesta presentada en este artículo contribuye significativamente a la descripción teórica sobre visualizaciones para el aprendizaje en ciencias, ya que los estudios previos se han concentrado sobre todo en imágenes de estructura simple; sin embargo, los textos escolares utilizan más bien estructuras múltiples que entremezclan lo narrativo y lo analítico. Asimismo, las investigaciones anteriores han indagado mínimamente en el rol del lenguaje verbal en ensambles predominantemente visuales. El artículo, por lo tanto, propone una taxonomía actualizada de ensambles multimodales que explican procesos científicos relevantes para el aprendizaje de la ciencia. Además, se utiliza la propuesta para describir dos ensambles multimodales sobre procesos químicos producidos por estudiantes de secundaria en el contexto de una tarea tipo examen. Este estudio visibiliza la importancia del lenguaje visual para el aprendizaje en las disciplinas y las diversas relaciones que establece con el lenguaje verbal en las prácticas de literacidad disciplinar.

Por último, Rosado, Mañas, Yúfera y Aparici analizan textos analíticos escritos en catalán por un grupo de estudiantes bilingües de catalán-español de secundaria y de primer año de universidad para identificar dificultades y trayectorias de desarrollo relativas a la conexión al interior de la oración y entre oraciones. Estudiaron el repertorio de marcas de conexión (conjunciones, marcadores parentéticos, marcadores léxicos) y las funciones que desempeñan (proposicional, estructural, modal). Los resultados muestran que los textos producidos por universitarios son más extensos en comparación con los de secundaria. Los y las estudiantes de secundaria también utilizan una proporción significativamente mayor de marcadores, con mayor frecuencia para las conjunciones. En cambio, en los textos universitarios, los conectores más frecuentes son los parentéticos. Se observa que escritores menos expertos incluyen conjunciones despojadas de su valor original para unir proposiciones, lo que ha sido reportado en otras investigaciones, aunque en otros géneros, en etapas más tempranas y típicamente en discursos orales. La presencia de marcadores proposicionales es significativamente mayor en el grupo de secundaria, mientras que en los modales, la relación se invierte. Mediante el análisis específico de los marcadores, se muestra que el compromiso de las y los escritores universitarios con su texto parece ser mayor. De esta manera, relacionar de manera competente y eficaz la información contenida en las oraciones o en unidades de discurso mayores en los textos analíticos parece constituir un aprendizaje que se prolonga incluso más allá de la adolescencia. De este artículo se infiere la importancia de la consideración de los géneros, específicamente de los recursos lingüísticos propios de aquellos analíticos, en el estudio del desarrollo tardío del lenguaje para promover prácticas pedagógicas que empoderen a las y los estudiantes en la toma de decisiones sobre los textos que construyen.

Del análisis de los artículos, resultan de interés las variadas formas de especificar el concepto de lenguaje a través de un adjetivo: por ejemplo, lenguaje académico, lenguaje argumentativo, lenguaje disciplinar. Todas estas distinciones dan cuenta del carácter situado del lenguaje y del acercamiento sociocultural para abordarlo con énfasis ya sea en el registro, en el género discursivo o en el campo disciplinar. También es posible identificar el uso indistinto entre lenguaje y lengua en estudios que enfatizan las diferencias según modalidad (oral y escrita). Por último, se encuentran estudios que destacan el rol de distintos modos semióticos -más allá del verbal- en la construcción de conocimientos en diversos contextos; de manera que se enfocan en el estudio del lenguaje visual o de la multimodalidad.

En algunos estudios, se distingue entre lenguaje y literacidad. En investigaciones de orientación psicológica, el término literacidad refiere a los procesos de aprendizaje escolar de la lectura y escritura, mientras que lenguaje alude más bien a la oralidad. En cambio, desde la propuesta sociocultural de los Nuevos Estudios de la Literacidad (Barton \& Hamilton, 2000), la literacidad es definida como una práctica social-mediada no solo por el lenguaje verbal sino también por otros modos semióticos- en la que diferentes actores participan y co-construyen significados tanto en espacios públicos como privados. De ahí que se diferencie, entonces, entre prácticas escolares entendidas como configuradoras de los sentidos al interior de las escuelas con fines pedagógicos y las vernáculas, definidas 
como aquellas acontecidas en los espacios locales e íntimos, animadas por objetivos personales y sin proceso formal de enseńanza. Esta diferencia, si bien es útil e importante para el estudio y la comprensión del desarrollo del lenguaje, no implica una dicotomía o una separación clara entre estos lenguajes, pues sus usos son concebidos más bien desde la lógica de un espiral progresivo desde prácticas del hogar, pasando por las escolares y llegando a las profesionales y ciudadanas (Uccelli et al., 2020). Como demuestran algunos de los estudios de este volumen, en el uso oral y escrito del lenguaje son las formas híbridas y multimodales las que priman y merecen cada vez mayor atención desde la investigación, así como un mayor aprovechamiento desde las prácticas docentes.

En cuanto al concepto de aprendizaje, los estudios no lo operacionalizan explícitamente, sino que más bien se infieren tres dimensiones para su abordaje. En primer lugar, se argumenta que el desarrollo del lenguaje oral sirve como precursor y continúa como proceso evolutivo conjunto y crucial que apoya el aprendizaje de la lectura y escritura, específicamente aquel que co-construye las prácticas escolares disciplinares. De ahí, entonces, el interés de comprender en mayor profundidad los recursos lingüísticos y discursivos necesarios para ser enseñados explícitamente en la escuela con el fin de que todas y todos los estudiantes puedan no solo acceder a los conocimientos construidos mediante este lenguaje, sino que también puedan producir géneros escolares analíticos. Es más, se espera que puedan también desarrollar habilidades metalingüísticas críticas que las y los animen a innovar e incluso transgredir normas estrictas o prescripciones en favor de la expresión personal, auténtica y reflexiva (Uccelli et al., 2020).

Los artículos se construyen sobre la premisa de que la escuela es un espacio para el aprendizaje de la lectura y escritura en el que el dominio del lenguaje contribuye al logro de este objetivo. Siguiendo esta misma línea de razonamiento, el lenguaje oral y escrito es comprendido como una herramienta mediadora de los aprendizajes. Entonces, una primera manera de entender el aprendizaje sigue una de las propuestas de Vygostky (1979) sobre mediación, quien plantea que el lenguaje no solo es un medio que permite la comunicación sino que también es una herramienta cultural que favorece la internalización de funciones cognitivas. En particular, Vygostky (1979) señala que el aprendizaje de la lengua escrita promueve el desarrollo de un razonamiento analítico, ya que aprender este sistema de signos permite la construcción de sentidos desvinculada del contexto físico inmediato de la comunicación.

En segundo lugar, se alude en términos genéricos a los aprendizajes que las y los estudiantes pueden adquirir si es que tienen un dominio del lenguaje escrito, analítico, académico o disciplinar. En otras palabras, los estudios apuntan a que el conocimiento del lenguaje oral y escrito facilita a las y los estudiantes el aprendizaje de distintos tópicos a los que pueden acceder a través de la lectura y escritura. Por lo tanto, estas son prácticas con potencial epistémico, pues promueven el aprendizajes de contenidos disciplinares (Serrano, 2014).

Por último, se amplía el campo del aprendizaje más allá de la institución escolar y se define específicamente el aprendizaje como una forma de estar en la sociedad, como práctica cultural y se propone el concepto de vidas de aprendizaje (learning lives) (Sefton-Green \& Erstad, 2017) para destacar el carácter constante, situado en múltiples contextos y abierto del aprender más allá de la escuela. En efecto, este concepto enfatiza cómo las personas navegan por espacios a través de los cuales constantemente aprenden en sus interacciones cotidianas; por ejemplo, en los espacios digitales se puede aprender a buscar contenidos a través de hashtags en español e inglés, a producir un dibujo tipo manga o a escribir una visión o misión de un colectivo, entre otros.

Teóricamente, estos estudios invitan a profundizar en conceptos que permitan superar la dicotomía entre lo oral y lo escrito para el aprendizaje escolar con el fin de poder comprender las múltiples relaciones entre modalidades, modos, registros, géneros y contextos. Ravid y Tolchinsky (2002) proponen el concepto de alfabetización lingüistica -linguistic literacy- para referirse al conocimiento que tienen los sujetos hablantes sobre sus repertorios lingüísticos, en parte producto de los procesos de aprendizaje de la lectura y escritura, para ponerlos en acto de manera consciente y flexible en géneros específicos. Por su parte, Uccelli y sus colegas (2020) presentan el concepto de lenguaje para la literacidad escolar-language for school literacy- para señalar tanto las 
prácticas como las habilidades discursivas que requieren aprender gradualmente las y los estudiantes para actuar de manera flexible en actividades de lectura y escritura en contextos de aprendizaje disciplinar. Estos conceptos muestran el dinamismo entre el lenguaje y el aprendizaje en la escuela, pero no incorporan aún el rol de otros modos semióticos y de otros espacios de aprendizaje no institucionalizados. Por lo tanto, se requiere de más teorización en el campo para mostrar las relaciones múltiples entre lenguajes, contextos y aprendizajes.

Los estudios de este número temático utilizan una miríada de perspectivas metodológicas, lo que da cuenta de la complejidad del problema educativo. Los aportes cuantitativos de corte más psicológico permiten entender las contribuciones de variables sociocognitivas y lingüísticas a los procesos de comprensión, así como determinar las diferencias entre grupos de estudiantes según sus preferencias por textos específicos. Los estudios que se enmarcan dentro del desarrollo tardío del lenguaje también utilizan métodos cuantitativos para determinar ya sea los efectos de intervenciones específicas con foco en las modalidades de la lengua o el uso de recursos específicos entre grupos. Un solo estudio cualitativo caracteriza las prácticas docentes para la implementación de un programa que promueve el análisis de textos de Historia. Desde una perspectiva más bien de análisis de corpus, el estudio sobre multimodalidad se enfoca en la caracterización de infografías en textos escolares de Ciencias. Por último, un estudio con elementos de la etnografía educativa y digital profundiza en las prácticas digitales de producción de jóvenes. Así, la relación entre lenguajes y aprendizajes va desde la generalización de los resultados hasta la comprensión en profundidad de múltiples asociaciones entre estos dos constructos.

Empíricamente, los estudios ofrecen evidencias para enriquecer una teoría sobre el aprendizaje del lenguaje y mediante el lenguaje con orientación pedagógica (Grøver et al., 2019). Todos los estudios presentan resultados que aportan significativamente al campo de investigación del desarrollo del lenguaje y de su enseñanza en la escuela, pero también especifican criterios para la implementación de prácticas pedagógicas con el fin de explicitar el rol de los lenguajes en la construcción de los aprendizajes. Dichas contribuciones se construyen desde una visión funcional y flexible del uso del lenguaje, así como desde la valoración de la diversidad lingüística y de los contextos de participación con propuestas específicas para apoyar a estudiantes y docentes más allá de una narrativa del déficit.

Este número temático de PEL fue diseñado desde Chile en tiempos de crisis y de transformación, tras una revuelta social que llevó a miles de personas a buscar algún modo - gritos, carteles, cacerolazos, rayados, canciones- para exigir dignidad y que tiene al país en medio de un proceso constituyente inédito en la historia. Fue pensado, escrito y deseado por autores y autoras de diversos lugares del mundo en medio de una pandemia que ha llevado a cuestionarse profundamente los sentidos de la educación y de la investigación. Esperamos que este número temático -Lenguajes para el aprendizaje-contribuya a la discusión sobre las múltiples relaciones entre lenguaje y aprendizaje considerando que el momento histórico en el que nos encontramos ha agrietado los espacios de lo conocido y ha potenciado el carácter fluido de los lenguajes. Sin duda, nuevas preguntas sobre las transformaciones sociales y culturales a partir de los lenguajes que co-construyen los aprendizajes surgen para la pospandemia, después de casi dos años en que se han incrementado las interacciones para el aprendizaje mediadas por lo digital. Lo que es más evidente ahora es que la investigación sobre el desarrollo del lenguaje tiene que dar cuenta, de alguna manera, del carácter cognitivo, sociocultural y político de los lenguajes y de los aprendizajes.

Financiamiento: Este número temático se ha desarrollado en el marco de los proyectos ANID/ CONICYT, FONDECYT 1190990 y FONDECYT 1200882, así como de la Beca de Doctorado Nacional ANID.

Agradecimientos: Queremos agradecer a las personas que revisaron y evaluaron los artículos por su enorme compromiso y su valiosísimo trabajo académico para contribuir al campo de la lingüística educacional. 


\section{Referencias}

Barton, D. \& Hamilton, M. (2000). Literacy practices. En D. Barton, M. Hamilton, \& R. Ivanič (Eds.), Situated literacies. Reading and writing in context (pp. 7-15). Londres, Inglaterra: Routledge.

Berman, R. \& Ravid, D. (2009). Becoming a literate language user: Oral and written text construction across adolescence. En D. Olson, \& N. Torrance (Eds.), The Cambridge handbook of literacy (pp. 92-111). Nueva York, NY: Cambridge University Press.

Buckingham, D. (2006). Defining digital literacy? What do you people need to know about digital media? En B. Bachmair (Ed.), Medienbildung in neuen Kulturräumen. VS Verlag für Sozialwissenschaften (pp. 59-71). https://doi.org/10.1007/978-3-531-92133-4_4

Burnett, C. \& Merchant, G. (2015). The challenge of 21st-Century Literacies. Journal of Adolescent \& Adult Literacy, 59(3), 271-274. https://doi.org/10.1002/jaal.482

Fang, Z., Schleppegrell, M. J. \& Cox, B. E. (2006). Understanding the language demands of schooling: Nouns in academic registers. Journal of Literacy Research, 38(3), 247-273. https://doi.org/10.1207/s15548430jlr3803_1

Graham, S. \& Harris, K. R. (2009). Almost 30 years of writing research: Making sense of it all with The Wrath of Khan. Learning Disabilities Research \& Practice, 24(2), 58-68. https://doi.org/10.1111/j.1540-5826.2009.01277.x

Grøver, V., Uccelli, P., Rowe, M. L. \& Lieven, E. (2019). Learning through language. En V. Grøver, P. Uccelli, M. L. Rowe, \& E, Lieven. (Eds.), Learning through language. Towards an educationally informed theory of language learning (pp. 1-16). Cambridge, Inglaterra: Cambridge University Press.

Macken-Horarik, M., Love, K., Sandiford, C. \& Unsworth, L. (2017). Functional grammatics. Re-conceptualizing knowledge about language and image for school English. Nueva York, NY: Routledge

Mills, K., Unsworth, L. \& Exley, B. (2018). Sensory literacies, the body and digital media. En K. Mills, A. Stornaiuolo, A. Smith, \& J. Zacher (Eds.), Handbook of writing, literacies, and education in digital cultures (pp. 26-36). Nueva York, NY: Routledge.

Nippold, M. A. (2007). Later language development: School-age children, adolescents, and young adults. Austin, TX: Pro-ed.

Ravid, D. \& Tolchinsky, L. (2002). Developing linguistic literacy: A comprehensive model. Journal of Child Language, 29(2), 419-448. https://doi.org/10.1017/S0305000902005111

Schleppegrell, M. (2001). Linguistic features of the language of schooling. Linguistics and Education, 12(4), 431-459. https://doi.org/10.1016/S0898-5898(01)00073-0

Schleppegrell, M. J. (2004). The language of schooling: A functional linguistic perspective. Londres, Inglaterra: Lawrence Erlbaum Associates.

Sefton-Green, J. \& Erstad, O. (2017). Researching “learning lives" -a new agenda for learning, media and technology. Learning, Media and Technology, 42(2), 246-250. https://doi.org/10.1080/17439884.2016.1170034

Serrano, S. (2014). La lectura, la escritura y el pensamiento. Función epistémica e implicaciones pedagógicas. Lenguaje, 42(1), 97-122. https://doi.org/10.25100/lenguaje.v42i1.4980

Snow, C. \& Uccelli, P. (2009). The challenge of academic language. En D. R. Olson \& N. Torrance (Eds.), The Cambridge handbook of literacy (pp. 112-133). Nueva York, NY: Cambridge University Press.

Uccelli, P. (2019). Learning the language for school literacy. En V. Grøver, P. Uccelli, M. L. Rowe, \& E. Lieven (Eds.), Learning through language. Towards an educationally informed theory of language learning (pp. 95-109). Cambridge, Inglaterra: Cambridge University Press.

Uccelli, P., Phillips Galloway, E., Barr, C. D., Meneses, A. \& Dobbs, C. L. (2015). Beyond vocabulary: Exploring crossdisciplinary academic-language proficiency and its association with reading comprehension. Reading Research Quarterly, 50(3), 337-356. https://doi.org/10.1002/rrq.104

Uccelli, P., Phillips Galloway, E. \& Qin, W. (2020). The language for school literacy: Widening the lens on language and reading relations. En E. B. Moje, P. Afflerbach, P. Enciso \& N. K. Lesaux (Eds.), Handbook of reading research (Vol. 5) (pp. 155-179). Nueva York, NY: Routledge.

Unsworth, L. \& Cleirigh, C. (2009). Multimodality and reading: The construction of meaning through image-text interaction. En C. Jewitt (Ed.), Handbook of Multimodal Analysis (pp. 151-164). Londres, Inglaterra: Routledge.

Vygotsky, L. (1979). El desarrollo de los procesos psicológicos superiores. Barcelona, España: Editorial Crítica. 